Extended abstract

\title{
Evolutionary relationships and genetic systems of rumen eubacteria
}

\author{
HJ Flint \\ Rowett Research Institute, Bucksburn, Aberdeen AB2I 9SB, UK
}

The mammalian rumen and the largely angiosperm plant material that is digested within it represent relatively recent developments in evolutionary history. On the other hand gut microbial ecosystems in general have been in existence for a much longer time, while cellulose and hemicellulose are ancient molecules present in cyanobacteria and algae. In trying to interpret the properties of present day rumen microorganisms we have to recognise that much of their evolutionary history involved responses to selection pressures quite different from those they encounter now [1,2]. Recruitment of species into the rumen microflora is assumed to have occurred progressively from other gut and non-gut habitats. It is expected that rapid coadaptation will have accompanied colonisation of the rumen. A striking example of this from the host side is the evolution of mammalian lysozyme, where rapid and convergent changes in the enzymes of ruminants and leaf eating colobine monkeys are assumed to represent adaptations to the recovery of microbial protein arising from pregastric fermentation $[3,4]$. We understand the coadaptive changes in the microbial symbionts far less well as yet. This is partly because of the bewildering diversity of organisms involved which is becoming ever more apparent both from conventional microbiology and from ribosomal RNA-based molecular analyses.

Currently recognised species such as Prevotella ruminicola and Butyrivibrio fibrisolvens include considerable interstrain genetic divergence $[5,6,7]$. Some of this diversity may reflect ancient divergence between groups that have shown little morphological and physiological change. However more detailed work is starting to reveal significant physiological differences; in the case of rumen Prevotella these have justified the definition of four new species, $P$. ruminicola (strain 23-like), P. bryantii (strain $\mathrm{B}_{1}$ 4-like), $P$. brevis (strain GA33like) and $P$. albensis (strain M384-like) [8]. Ribosomal RNA-based analyses have suggested that these species generally coexist in the rumens of many different animals indicating that they probably occupy different niches in the system [9]. 
Evolution of polysaccharide degrading enzyme systems - a story of multigene families

Apart from ribosomal RNA studies, most information relevant to evolutionary change in rumen bacteria at the molecular level has inevitably come from the study of plant cell wall polysaccharidase genes. It is clear that gene duplication has played a major role in generating enzyme diversity resulting in complex multigene families which reflect both ancient and comparatively recent duplication events. In addition polysaccharidases generally show a modular structure resulting from fusions between different domains. A good example is provided by four xylanases of Ruminococcus flavefaciens 17 which are all related at their amino termini by family 11 catalytic domains $[10,11]$. Interestingly three of these enzymes also carry a noncatalytic region that resembles thermostabilising domains found linked to family 10 xylanases in thermophilic eubacteria [12]. It seems likely that these 'stabilising' domains have evolved to fulfil a different role in the mesophilic rumen species, particularly in view of the fact that they are linked to a different type of xylanase. A second non-catalytic region present in two of these multidomain xylanases was recently found to be present also in a cellulase from R. flavefaciens 17 [13]. This region shows significant similarity with dockerin sequences from cellulolytic Clostridium spp implying that these enzymes form part of a large multienzyme complex on the cell surface. At least five $R$. flavefaciens cellulases ranging from $70-120 \mathrm{kDa}$ were found among proteins that bound to cellulose [11]. While there is also evidence for non-cellulosomal enzymes, these com- plexes are assumed to play a vital role in the concerted attack on the diverse, closely associated polysaccharides present in plant cell walls. A cluster of genes required for the utilization of xylan degradation products has also been found in $R$. flavefaciens 17 . Hybridisation experiments indicate that this cluster, which includes xylose isomerase, is present in other xylanutilising $R$. flovefaciens strains, but is absent from strains that cannot utilise xylan degradation products. Interestingly one of the genes of the cluster shows homology with family $3 \beta$-glucosidases, but appears to resemble the Trichoderma reesei $\mathrm{BXI} 1$ enzyme [14] in showing $\beta$-xylosidase rather than $\beta$-glucosidase activity. The $R$. flavefaciens enzyme differs from two other family $3 \beta$-glucosidases reported from rumen bacteria, from $B u$. fibrisolvens and $R$. albus, which are unusual in showing a rearrangement of $\mathrm{N}$ and $\mathrm{C}$ terminal regions compared with other members of this family [15]. We might argue from this that either the $R$. flavefaciens or the $R$. albus genes were acquired by horizontal transfer. A more likely explanation, however, is that two distinct subfamilies of family $3 \beta$-glucosidases have become specialised for different functions, and that representatives of both subfamilies are present in many Gram-positive rumen bacteria.

Xylan-degrading enzymes of Prevotella spp show a very different organisation and structure, which is assumed to be geared to the efficient utilization of xylan breakdown products. There is evidence for $P$. ruminicola and $P$. bryantii that most $\mathrm{xy}$ lanolytic activity is present within the cell, and is concerned with processing of $\mathrm{xy}-$ looligosaccharides. In $P$. bryantii a gene cluster $(x y n A, B)[16]$ encodes an exoxyla- 
nase and an endoxylanase that shows great similarity to the $x y l I / x s a$ cluster [17] present in the xylanolytic human hind gut species Bacteroides ovatus. The cloned $P$. bryantii exoxylanase showed considerable oxygen sensitivity when expressed in Escherichia coli, but was far less sensitive in intact cells of $E$. coli or B. vulgatus. The normal cellular location of this enzyme and the anaerobic environment of the rumen presumably protect it against oxygen damage. The genetic divergence between $P$. ruminicola and $P$. bryantii is such that we could not detect any cross hybridisation between cloned xylanases from the two species. Nevertheless the predicted structures of the major SDS-renaturable xylanases $(70 \mathrm{kDa}$ in $P$.bryantii and $80 \mathrm{kDa}$ in P.ruminicola) show that they are both family 10 xylanases containing unique interrupting sequences within their catalytic domains. Phylogenetic analyses indicate that these interrupting regions, which are unrelated in sequence but show the same site of insertion in the two enzymes, arose from independent insertions into a common ancestral gene [18]. It seems likely that these insertions affect the catalytic properties of the enzyme, and the inserted XynC enzyme shows a preference for larger substrates ( $>\mathrm{X} 8)$ than the uninserted XynA. Sequence comparisons indicate that the inserted family 10 enzymes represent a divergent subgroup of this family that may have evolved to fulfil a distinct role.

No family 11 xylanase has so far been isolated from a Prevotella sp., although such domains are common to all known Ruminococcus xylanases, as discussed earlier, and are also reported from Fibrobacter succinogenes and from rumen fungi. The possibility exists that Prevotella sp., or perhaps members of the Cytophaga
- Flexibacter - Bacteroides phylum as a whole, may not have acquired family 11 enzymes. It is not obvious what consequences this would have for the ability to degrade xylans, but we found no evidence that pretreatment of xylans with a cloned family 11 xylanase from $R$. flavefaciens increased overall pentose utilization from xylan by $P$. bryantii $\mathrm{B}_{1} 4$ cultures [19].

In conclusion, while a few related catalytic domains responsible for cleaving particular glycosidic bonds are quite widely distributed, the organisation of these domains into enzymes and of enzymes into enzyme complexes varies widely according to the species and the niche it occupies. This raises fundamental questions. To what extent have the degradative capacities of individual rumen organisms been limited by a failure to acquire a few key degradative genes? Can enzyme systems effective in plant cell wall degradation be produced other than by a protracted process of coadaptation involving large numbers of genes? The answers depend in part on the role of horizontal gene transfer in the evolution of rumen organisms. While sequence data can potentially provide information about horizontal gene flow, they must be interpreted with caution particularly when dealing with multigene families. As noted above, where enzyme phylogenies initially appear to be discordant with 16SrRNA relationships these discordancies may disappear if it is later found that comparisons have involved members of different subfamilies. The distribution of certain polysaccharidase gene families is suggestive of horizontal gene flow between ancestors of ruminal bacteria and fungi [20] but provides no evidence that such transfer occurred in the rumen. Such transfer events may often have predated the recruitment 
of the species to the rumen flora. Direct experimental evidence of gene flow between rumen bacteria is considered below.

\section{Natural mechanisms of gene transfer among rumen bacteria}

The high density and diversity of the rumen microbial ecosystem, together with the existence of special microhabitats associated with substrate particles and protozoal food vacuoles, suggests that it could be an extremely active site for gene transfer both within and between species $[21,22]$. Although it has been widely assumed that horizontal gene transfer must occur, there is little experimental evidence. Plasmids have been reported from many rumen bacteria and among obligate anaerobes the only associated functions so far detected are antibiotic resistance genes $[23,24]$. Only in Bu. fibrisolvens [25] and Selenomonas ruminantium [26] have plasmids been found that are large enough to carry significant amounts of information beyond the genes necessary for their own replication and maintenance. Plasmid transfer in the presence of a complete rumen flora has been demonstrated only for rumen $E$. coli [27]. Recent work has demonstrated natural, high frequency transfer of a tetracycline resistance determinant between strains of $B u$. fibrisolvens, which has been shown to involve a novel chromosomal element estimated to be $50-60 \mathrm{~kb}$ in size that has a prefered insertion site in the recipient chromosome [28]. Several $\mathrm{Bu}$. fibrisolvens strains were found to harbour a second $\mathrm{Tc}^{\mathrm{R}}$ gene related to TetM for which transfer was not detected. Thus in addition to transmissible elements, it is likely that certain elements have been acquired that cannot be further transferred. In $P$. ruminicola although TetQ is en- coded by a self transmissible plasmid in one strain [23] there is evidence that in other rumen strains it may be linked to sequences showing homology with a transmissible Bacteroides chromosomal element [29]. Given the importance of conjugal chromosomal elements in Bacteroides and in Gram-positive bacteria $[30,31]$ it seems likely that such elements will be found increasingly in rumen bacteria, most of which are related to these groups. A potentially significant feature is the ability to mobilise other genetic elements, a property also shown by a chromosomal sex factor identified in lactococci [32]. The abundance of rumen bacteriophage $[33,34]$ suggests that phage mediated transfer may also be a significant mechanism. The extent of genetic interchange between the strict anaerobes of the rumen and the microflora of the hind gut of animals and man is still largely an open question, although significant sequence similarity and genetic exchange has been found between TetQ determinants of rumen and human origin in Bacteroides/Prevotella spp [35]. Our limited understanding of genetic diversity in these groups, discussed already, means that we still do not know to what extent closely related strains of obligate anaerobes can colonise different host species.

Understanding these aspects of natural gene transfer and evolution in rumen microorganisms is not of purely academic interest. This understanding should help us to predict the likely effects of transferring novel genes into alternative rumen species and should also suggest new approaches to the manipulation of rumen function. It is also relevant to the possible role of the rumen flora in disseminating antibiotic resistances and, in future, transgenes in the environment. The widespread use of 
transgenic plants and the successful construction of manipulated rumen and silage microorganisms (36) requires that we learn a great deal more about ruminal gene transfer.

1. Woese CR (1987) Microbiol Rev 51, 221-271

2. Ochman H, Wilson AC (1987) $\mathrm{J} \mathrm{Mol}$ Evol 26, 74-86

3. Stewart C-B, Schilling JW, Wilson AC (1987) Nature 330, 401-404

4. Messier W, Stewart C-B (1997) Nature 385, 151-154

5. Willems AM, Collins MD (1996) Int $J$ Syst Bacteriol 46, 195-199

6. Forster RJ, Teather RM, Gong J, Deng S-J (1996) Lett Appl Microbiol 23, 218 222

7. Avgustin G, Wright F, Flint HJ (1994) Int J Syst Bacteriol 44, 246-255

8. Avgustin G, Wallace RJ, Flint HJ (1997) Int J Syst Bacteriol 47, 284-288

9. Wood J, Avgustin G, Scott KP, Flint HJ (1995) In: Beijerinck Centennial Meeting, The Hague 100-101

10. Flint HJ, Forsberg CW (1995) In: Ruminant Physiology: Digestion, Metabolism, Growth and Reproduction. Proceedings of the Eigth International Symposium on Ruminant Physiology, (Engelhardt WV, Leonhard-Marek S, Breves G, Giesecke D eds) Ferdinand Enke Verlag Stuttgart, 43-70

11. Kirby J (1997) PhD Thesis, University of Aberdeen

12. Winterhalter $\mathrm{C}$, Heinrich $\mathrm{P}$, Candussio $\mathrm{A}$, Wich G, Liebl, W (1995) Mol Microbiol 15, 431-444

13. Kirby J, Martin J, Daniel AS, Flint HJ (1997) FEMS Microbiol Lett 149, 213-219

14. Margolles-Clark E, Tenkanen M, NakariSetala T, Pentilla, M (1996) Appl Environ Microbiol 62, 3840-3846

15. Rojas A, Romeu A (1996) FEBS lett 378, 93-97
16. Gasparic A, Martin JC, Daniel AS, Flint HJ (1995) Appl Environ Microbiol 61, 2958-2964

17. Whitehead TR (1995) Biochim Biophys Acta 1244, 239-241

18. Flint HJ, Whitehead TR, Martin J, Gasparic A (1997) Biochim Biophys Acta 1337, 161-165

19. Miyazaki K, Martin JC, Marinsek-Logar $\mathrm{R}$ Flint, $\mathrm{HJ}$ Anaerobe (in press)

20. Syvanen M (1994) Annu Rev Genet 28, 237-261

21 Flint HJ (1994) FEMS Microbiol Lett 121, 259-268

22 Morrison M (1996) J Dairy Sci 79, 1476-1486

23. Flint HJ, Thomson AM, Bisset, J (1988) Appl Environ Microbiol 54, 855-860

24. Ohmiya K, Hoshino C, Shimizu S (1989) Asian Australas J Anim Sci 2, 501-502

25. Teather RM (1982) Appl Environ Microbiol 43, 298-302

26. Fliegorova K, Benada O, Flint HJ (1997) Lett Appl Microbiol (in press)

27. Scott KP, Flint HJ (1995) J Appl Bacteriol 78, 189-193

28. Scott KP, Barbosa TM, Forbes K, Flint HJ (1997) Appl Environ Microbiol 63, 3405-3411

29. Nikolich MP, Shoemaker NB, Wang GR, Salyers AA (1994) J Bacteriol 176, 6606-6612

30. Clewell DB, Flanagan SE, Jaworski DD (1995) Trends Microbiol 3, 229-236

31. Salyers AA, Shoemaker NB, Stevens AM, Li L-Y (1995) Microbiol Rev 59, 579-590

32. Gasson MJ, Swindell S, Maeda S, Dodd HM (1992) Mol Microbiol 6, 3213-3223

33. Selinger LB, Forsberg CW, Cheng $\mathrm{KJ}$ (1996) Anaerobe 2, 263-284

34. Klieve AV, Swain RA (1993) Appl Environ Microbiol 59, 2299-2303

35. Nikolich MP, Hong G, Shoemaker N, Salyers AA (1994) Appl Environ Microbiol 60, 3255-3260 
36. Gregg K, Cooper CL, Schafer DJ, Sharpe H, Beard CE, Allen G, Xu J 